\title{
Estudo de sinterização do compósito $\mathrm{Si}_{3} \mathrm{~N}_{4} / \mathrm{TiC}$
}

\section{(Sintering studies of the $\mathrm{Si}_{3} \mathrm{~N}_{4} / \mathrm{TiC}$ composite)}

\author{
A. C. S. Coutinho, J. C. Bressiani, A. H. A. Bressiani \\ Instituto de Pesquisas Energéticas e Nucleares \\ Av. Prof. Lineu Prestes, 2242, Cidade Universitária, S. Paulo, SP 05508-000
}

\begin{abstract}
Resumo
A busca por melhores propriedades mecânicas e maior confiabilidade de cerâmicas à base de $\mathrm{Si}_{3} \mathrm{~N}_{4}$ faz com que seus compósitos sejam desenvolvidos e intensivamente estudados. A maioria das pesquisas realizadas foca as interações e propriedades mecânicas obtidas, mas interações químicas entre as fases foram pouco estudadas. O objetivo deste trabalho é estudar as interações químicas que ocorrem durante a sinterização do compósito $\mathrm{Si}_{3} \mathrm{~N}_{4}-20 \%$ vol. TiC. A cinética de sinterização foi estudada por dilatometria, com patamar a $1750{ }^{\circ} \mathrm{C} / 1 \mathrm{~h}$ em atmosfera dinâmica de $\mathrm{N}_{2}$. Os resultados de dilatometria indicam alterações na cinética de sinterização, quando comparado ao $\mathrm{Si}_{3} \mathrm{~N}_{4}$ monolítico. Para avaliar as fases que se formam em temperaturas intermediárias, os compósitos foram tratados termicamente entre $1400{ }^{\circ} \mathrm{C}$ e $1850{ }^{\circ} \mathrm{C}$. As amostras foram caracterizadas por difração de raios X. Foi observada a formação da solução sólida TiCN e da fase SiC.
\end{abstract}

Palavras-chave: nitreto de silício, sinterização, compósitos, TiC .

Abstract

During the last years much attention was given towards the better properties and reliability of $\mathrm{Si}_{3} \mathrm{~N}_{4}$ based composites. Most of the research focus on the improvement of the mechanical properties but not on the chemical interaction between the phases of the composite. The purpose of this work is to study the chemical interactions between $\mathrm{Si}_{3} \mathrm{~N}_{4}$ and TiC during the sintering process. The kinetics of sintering was studied by means of dilatometry, at $1750{ }^{\circ} \mathrm{C} / 1 \mathrm{~h}$ in a dynamic atmosphere of $\mathrm{N}_{2}$. Thermal treatments were carried out in the temperature range $1400-1850{ }^{\circ} \mathrm{C}$ to analyze the reactions. The phases were determined by $X$-ray diffraction. Alterations on the sintering kinetics due to the presence of the second phase were observed. These alterations must be related to the formation of the TiCN solid solution and SiC, observed in X-ray diffraction.

Keywords: silicon nitride, sintering, composite, TiC.

\section{INTRODUÇÃO}

$\mathrm{O} \mathrm{Si}_{3} \mathrm{~N}_{4}$ possui papel fundamental dentre as cerâmicas para fins estruturais, principalmente devido à sua boa resistência ao choque térmico e alta resistência à corrosão. $\mathrm{O}$ nitreto de silício também apresenta boa estabilidade química, boa resistência à abrasão e baixo coeficiente de expansão térmica. Esta combinação de propriedades faz com que seja um material adequado para aplicações em condições extremas de temperatura e solicitações mecânicas [1].

A confiabilidade do $\mathrm{Si}_{3} \mathrm{~N}_{4}$ pode ser melhorada pela adição de reforços na forma de whiskers, fibras ou partículas formando compósitos [2]. Os compósitos à base de nitreto de silício têm sido bastante estudados e dentre estes compósitos o mais comum é o compósito com adição de $\mathrm{SiC}$, como fase reforçante [3].

O principal objetivo das pesquisas relacionadas aos compósitos é melhorar as propriedades mecânicas do material [4], mas as interações químicas que podem ocorrer

Trabalho apresentado no $48^{\circ}$ Congresso Brasileiro de Cerâmica, Curitiba, PR, 2004. entre o nitreto de silício e a segunda fase precisam ser [4] já que durante o processamento dos materiais podem ocorrer interações entre as fases, modificar a cinética de sinterização, influenciar a densificação do material e produzir um efeito contrário ao desejado em relação às propriedades mecânicas finais do compósito [3].

O processo de sinterização do nitreto de silício é bastante complexo e ocorre via fase líquida. Devido a natureza covalente das ligações químicas entre os átomos de $\mathrm{Si}$ e $\mathrm{N}$, o coeficiente de auto difusão é baixo, sendo necessário o uso de aditivos de sinterização que formem um líquido adequado durante a sinterização e permita que o processo de solução-reprecipitação ocorra. Os aditivos mais comuns são os óxidos metálicos e de terras raras, como $\mathrm{Al}_{2} \mathrm{O}_{3}, \mathrm{Y}_{2} \mathrm{O}_{3}, \mathrm{Ce}_{2} \mathrm{O}_{3}$ e $\mathrm{La}_{2} \mathrm{O}_{3}$ [5].

Os aditivos de sinterização reagem com a sílica presente na superfície do pó de $\mathrm{Si}_{3} \mathrm{~N}_{4}$, formando um líquido, que auxilia no rearranjo das partículas, no primeiro estágio da sinterização. Com o aumento da temperatura, a fase $\alpha$ $\mathrm{Si}_{3} \mathrm{~N}_{4}$ se solubiliza no líquido formado pelos aditivos de sinterização e quando este líquido se torna supersaturado, inicia-se a reprecipitação, na forma de $\beta-\mathrm{Si}_{3} \mathrm{~N}_{4}$ [6-8]. 
A presença de partículas de segunda fase pode provocar alterações na sinterização do $\mathrm{Si}_{3} \mathrm{~N}_{4}$, dificultar o rearranjo das partículas, o transporte de massa e reagir quimicamente com os constituintes do sistema $[8,9]$

Poucos trabalhos foram publicados envolvendo as interações químicas entre o $\mathrm{Si}_{3} \mathrm{~N}_{4}$ e o TiC. Em [10] foi apresentado um estudo das interações entre o $\mathrm{Si}_{3} \mathrm{~N}_{4}$ e o $\mathrm{TiC}$ e o equilíbrio termodinâmico entre as fases. A partir dos resultados obtidos, os autores propuseram um diagrama de estabilidade dos componentes deste sistema. A pressão parcial de nitrogênio durante a sinterização influencia a termodinâmica de formação das fases, como o TiCN e o SiC.

Em compósitos SiAlON-TiC foi observado que ocorrem reações semelhantes às observadas no compósito com $\mathrm{Si}_{3} \mathrm{~N}_{4}$, formando $\mathrm{TiCN}$ e $\mathrm{SiC}$ [11].

O objetivo deste trabalho é estudar as interações químicas que ocorrem entre o $\mathrm{Si}_{3} \mathrm{~N}_{4}$ e o TiC durante a sinterização, a cinética de sinterização e analisar os produtos de reação.

\section{MATERIAIS E MÉTODOS}

Os compósitos foram preparados pela rota tradicional de preparação de pós. Uma composição contendo $90 \%$ em peso de $\alpha-\mathrm{Si}_{3} \mathrm{~N}_{4}$ (M11-H.C. Starck, 92,7\% de $\alpha-\mathrm{Si}_{3} \mathrm{~N}_{4}$ ) e como aditivos de sinterização $6 \%$ em peso de $\mathrm{Y}_{2} \mathrm{O}_{3}$ (Aldrich Chemical) e $4 \%$ em peso de $\mathrm{Al}_{2} \mathrm{O}_{3}$ (A16/SG - Alcoa) foi preparada. Esta composição foi misturada em moinho de alta energia, por $4 \mathrm{~h}$, rotação $300 \mathrm{rpm}$ e meio líquido álcool isopropílico. A suspensão obtida foi seca em roto-evaporador e o pó desaglomerado em malha ASTM 80. Esta composição é denominada CB.

O compósito foi preparado seguindo a mesma rota de preparação utilizada para a preparação da CB. À composição CB foi adicionado $20 \%$ em volume de TiC (H.C. Starck, com 1100 ppm de carbono livre). A suspensão obtida foi seca em roto-evaporador e após a desaglomeração, os pós foram

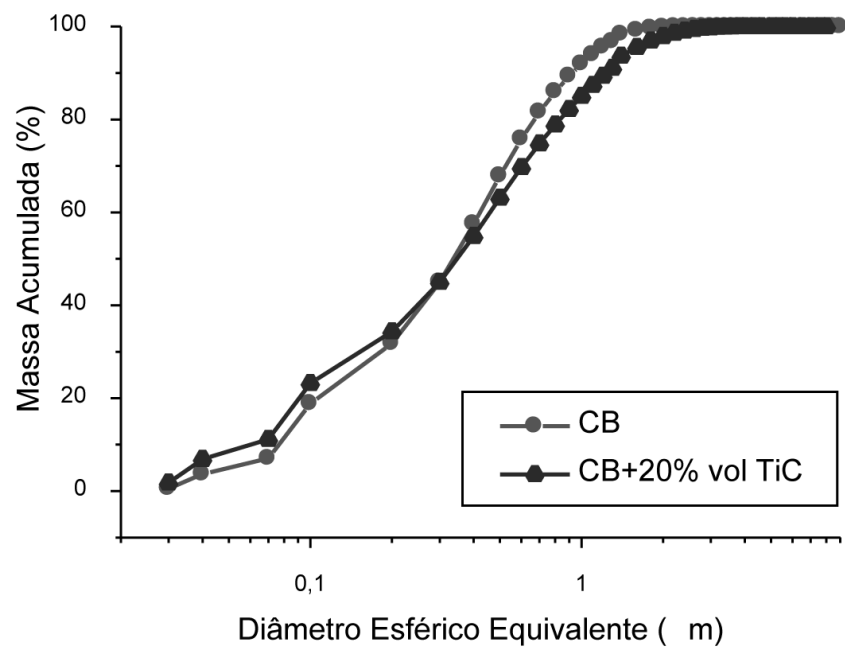

Figura 1: Distribuição granulométrica das composições em estudo. [Figure 1: Particle size distribution of the compositions.]

compactados por prensagem uniaxial (50 MPa) e isostática à frio $(200 \mathrm{MPa})$. Os pós foram caracterizados quanto à distribuição de tamanho de partícula. A densidade à verde das amostras foi determinada pelo método geométrico.

Para o estudo de cinética de sinterização, uma amostra com $7 \mathrm{~mm}$ de diâmetro foi sinterizada em dilatômetro (Netzsch modelo DIL $402 \mathrm{E} / 7$ ) a $1750{ }^{\circ} \mathrm{C}$, por $1 \mathrm{~h}$, com taxa de aquecimento e resfriamento de 20 e $30^{\circ} \mathrm{C} / \mathrm{min}$, respectivamente. Foram utilizados tubo e haste de contato de grafite e fluxo de $\mathrm{N}_{2}$

Para analisar as fases formadas durante a sinterização do compósito, foram realizados tratamentos térmicos em diferentes temperaturas em forno de resistência de grafite (Thermal Technologies, Santa Barbara, USA) a $1400^{\circ} \mathrm{C} / 1 \mathrm{~h}$, $1500^{\circ} \mathrm{C} / 1 \mathrm{~h}, 1600^{\circ} \mathrm{C} / 1 \mathrm{~h}, 1700{ }^{\circ} \mathrm{C} / 1$ h e $1750{ }^{\circ} \mathrm{C} / 1 \mathrm{~h}+1850^{\circ} \mathrm{C} / 1 \mathrm{~h}$, em atmosfera de $\mathrm{N}_{2}$.
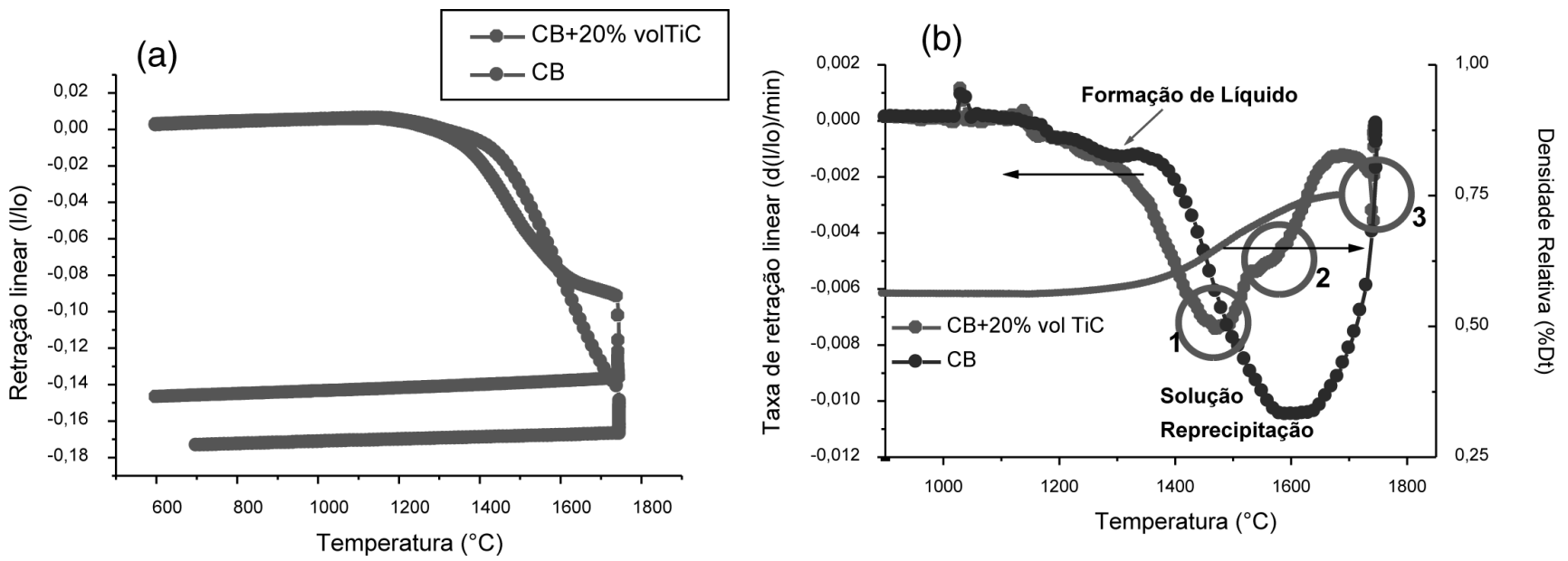

Figura 2: Curvas de (a) retração linear e (b) taxa de retração linear e densidade relativa em função da temperatura.

[Figure 2: Results of dilatometric experiments: (a) linear shrinkage vs. temperature and (b) shrinkage rate vs. temperature.] 
A densidade das amostras foi determinada pelo método de imersão e as fases cristalinas por difração de raios X.

\section{RESULTADOS E DISCUSSÃO}

A distribuição granulométrica das misturas foi obtida por espalhamento a laser. É observada distribuição homogênea e diâmetro médio de partículas de aproximadamente $0,30 \mu \mathrm{m}$.

A densidade teórica foi calculada de acordo com a regra das misturas e a densidade a verde das amostras é aproximadamente 55\% da densidade teórica.

Com os dados obtidos durante a sinterização assistida por

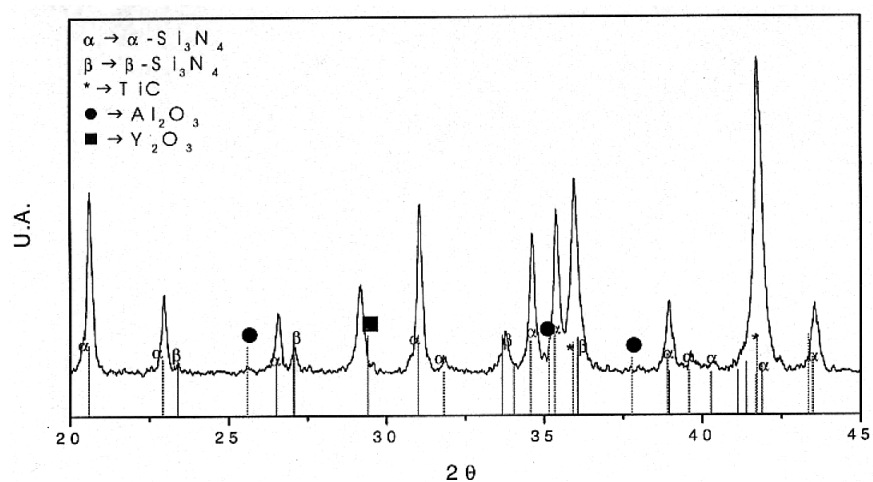

Figura 3: Difração de raios $X$ da mistura $\mathrm{CB}+20 \%$ vTiC.

[Figure 3: X-ray diffraction patterns of the starting powder.]
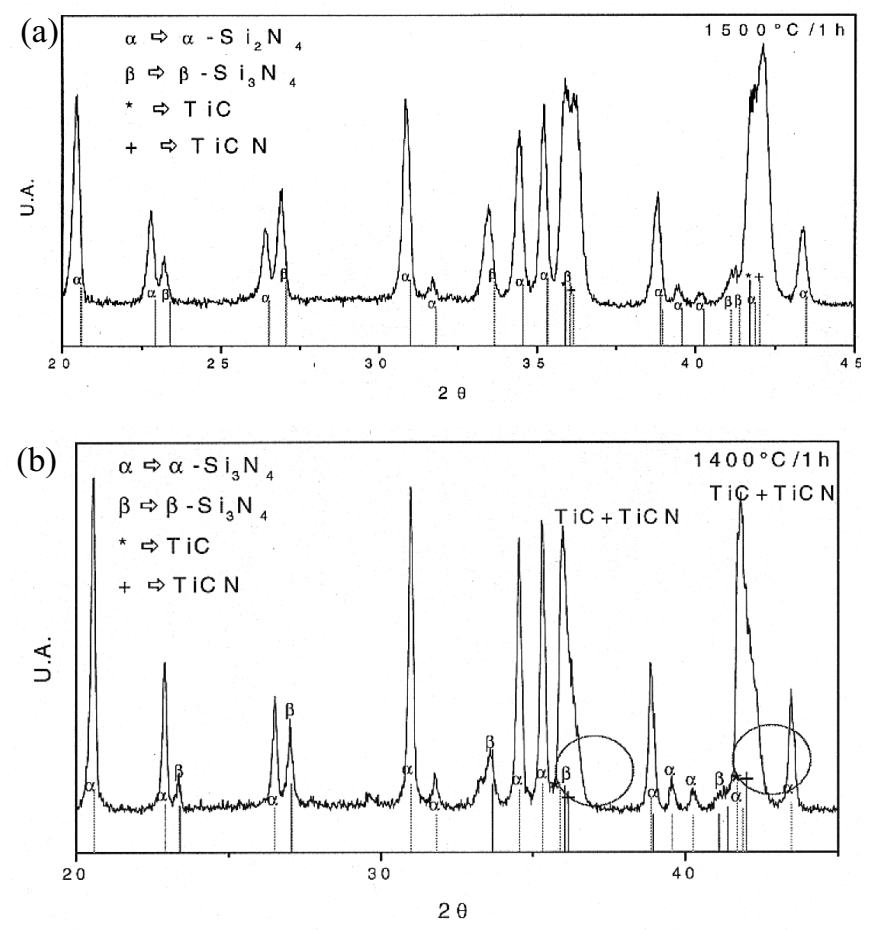

Figura 4: Difratogramas de raios $\mathrm{X}$ do compósito $\mathrm{CB}+20 \%$ vol TiC; (a) $1400{ }^{\circ} \mathrm{C} / 1 \mathrm{~h}$; (b) $1500{ }^{\circ} \mathrm{C} / 1 \mathrm{~h}$.

[Figure 4: $X$-ray diffraction patterns of the composite $C B+20$ vol\% $\mathrm{TiC}:$ (a) $1400{ }^{\circ} \mathrm{C} / 1 \mathrm{~h}$; (b) $1500{ }^{\circ} \mathrm{C} / 1 \mathrm{~h} . \mathrm{J}$ dilatômetro foram traçadas curvas de retração linear, taxa de retração linear e densidade relativa em função da temperatura (Fig. 2).

A curva de retração linear do material compósito apresenta comportamento semelhante à curva da $\mathrm{CB}$ e as duas apresentam retração durante o patamar. $\mathrm{O}$ início da retração ocorre aproximadamente na mesma temperatura $\left(\sim 1200{ }^{\circ} \mathrm{C}\right)$, mas a retração total observada do compósito é inferior à observada na CB.

$\mathrm{Na}$ curva de taxa de retração linear em função da temperatura da amostra CB e do compósito é observada uma alteração na taxa de retração linear (pico) em aproximadamente $1200{ }^{\circ} \mathrm{C}$ (Fig. 2 b), devido à formação de líquido e rearranjo das partículas. Com o aumento da
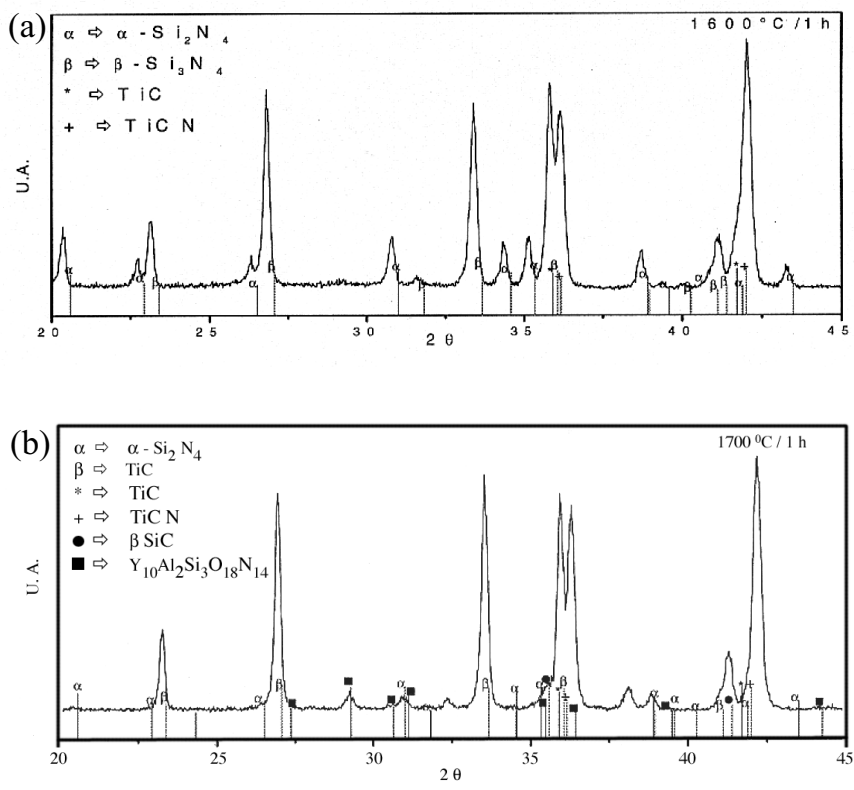

Figura 5: Difratogramas de raios $\mathrm{X}$ do compósito $\mathrm{CB}+20 \%$ vol TiC: (a) $1600{ }^{\circ} \mathrm{C} / 1 \mathrm{~h}$; e (b) $1700{ }^{\circ} \mathrm{C} / 1 \mathrm{~h}$.

[Figure 5: X-ray diffraction patterns of the composite CB+20vol\%TiC: (a) $1600{ }^{\circ} \mathrm{C} / 1 \mathrm{~h}$; (b) $\left.1700{ }^{\circ} \mathrm{C} / 1 \mathrm{~h}.\right]$

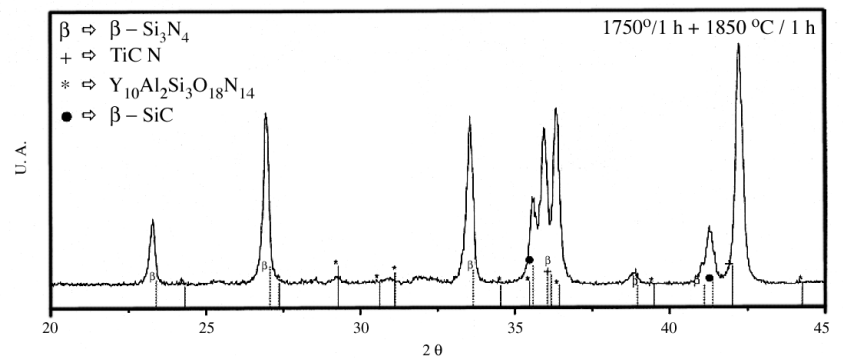

Figura 6: Difratogramas de raios $\mathrm{X}$ do compósito $\mathrm{CB}+20 \%$ vol TiC: $1750{ }^{\circ} \mathrm{C} / 1 \mathrm{~h}+1850{ }^{\circ} \mathrm{C} / 1 \mathrm{~h}$.

[Figure 6: X-ray diffraction patterns of the composite CB+20vol\%TiC: (a) $\left.1750{ }^{\circ} \mathrm{C} / 1 \mathrm{~h}+1750{ }^{\circ} \mathrm{C} / 1 \mathrm{~h}.\right]$ 
temperatura, tem início o processo de solução-reprecipitação da $\mathrm{CB}$, com máxima taxa de retração em aproximadamente $1600{ }^{\circ} \mathrm{C}$. Na curva de taxa de retração linear em função da temperatura do compósito são observados três picos (pico 1, pico 2 e pico 3 - Fig. 2 b) referentes a alterações na taxa de retração linear. Essas alterações devem estar relacionadas com a presença de TiC e com interações químicas entre os constituintes.

Para estudar a alteração observada na taxa de retração linear do compósito, foram realizados tratamentos térmicos em diferentes temperaturas $\left(1400{ }^{\circ} \mathrm{C} / 1 \mathrm{~h}, 1500{ }^{\circ} \mathrm{C} / 1 \mathrm{~h}\right.$, $1600^{\circ} \mathrm{C} / 1 \mathrm{~h}, 1700^{\circ} \mathrm{C} / 1 \mathrm{he} 1750^{\circ} \mathrm{C} / 1 \mathrm{~h}+1850^{\circ} \mathrm{C} / 1 \mathrm{~h}$ ) e após cada tratamento térmico as fases presentes foram determinadas por difração de raios X. Os difratogramas são apresentados a seguir (Fig. 4, Fig. 5 e Fig. 6). O difratograma da mistura inicial é apresentado na Fig. 3.

Observa-se na mistura inicial apenas as fases $\alpha$ e $\beta-\mathrm{Si}_{3} \mathrm{~N}_{4}$, a fase $\mathrm{TiC}$ e os aditivos de sinterização $\mathrm{Al}_{2} \mathrm{O}_{3}$ e $\mathrm{Y}_{2} \mathrm{O}_{3}$. Não são encontradas evidências de que reações ocorram durante o processamento.

Após o tratamento a $1400{ }^{\circ} \mathrm{C}$, adicionalmente aos picos de $\alpha-\mathrm{Si}_{3} \mathrm{~N}_{4}$, são observados os picos de $\beta-\mathrm{Si}_{3} \mathrm{~N}_{4}$, indicando que o processo de transformação de fase já teve início. Os picos referentes à fase $\mathrm{TiC}$ apresentam alargamento em sua base (enfatizado no difratograma) indicando a formação de uma solução sólida. A solução sólida que se forma é TiCN, observada nos difratogramas referentes aos tratamentos em maior temperatura. Nos difratogramas referentes aos tratamentos a $1400{ }^{\circ} \mathrm{C}$ e $1500{ }^{\circ} \mathrm{C}$, observa-se a presença do pico referente à fase $\mathrm{TiC}$ e à fase $\mathrm{TiCN}$, e após os tratamentos a $1700{ }^{\circ} \mathrm{C}, 1750{ }^{\circ} \mathrm{C}+1850{ }^{\circ} \mathrm{C}$ a fase TiC não é mais observada, indicando que todo o $\mathrm{TiC}$ reagiu para formar a solução sólida TiCN.

Segundo a literatura, a fase TiCN é formada a partir de $1100{ }^{\circ} \mathrm{C}$, na presença de $\mathrm{TiC}$ e $\mathrm{N}$ [4]. Durante o tratamento térmico foi utilizada atmosfera de $\mathrm{N}_{2}$ e a reação pode estar ocorrendo por reação do $\mathrm{N}_{2}$ da atmosfera com o TiC.

O TiC é um composto não estequiométrico com estrutura cristalina do tipo $\mathrm{NaCl}$, onde os átomos de Ti ocupam as posições octaédricas. A formação da fase TiCN envolve difusão em estado sólido, onde os átomos de $\mathrm{C}$, da rede do TiC, são substituídos por átomos de $\mathrm{N}$, formando carbonitreto de titânio, de acordo com a reação [10]:

$$
\mathrm{TiC}_{(\mathrm{s})}+\frac{\mathrm{x}}{2} \mathrm{~N}_{2(\mathrm{~g})} \rightarrow \mathrm{TiC}_{1-\mathrm{x}} \mathrm{N}_{\mathrm{x}(\mathrm{s})}+\mathrm{xC}_{(\mathrm{s})}
$$

Após os tratamentos a $1700{ }^{\circ} \mathrm{C}$ e a $1750{ }^{\circ} \mathrm{C}+1850{ }^{\circ} \mathrm{C}$ foi identificada a fase $\mathrm{SiC}$. A presença de $\mathrm{SiC}$ só pode estar associada à decomposição do $\mathrm{Si}_{3} \mathrm{~N}_{4}$. Nas condições dos tratamentos térmicos, a decomposição do $\mathrm{Si}_{3} \mathrm{~N}_{4}$ deve ter sido favorecida [10]. Durante os tratamentos térmicos a pressão foi de $\sim 80$ psi ( $~ 5$ atm) e portanto a formação de SiC está de acordo com o diagrama termodinâmico proposto na literatura (Fig. 7).

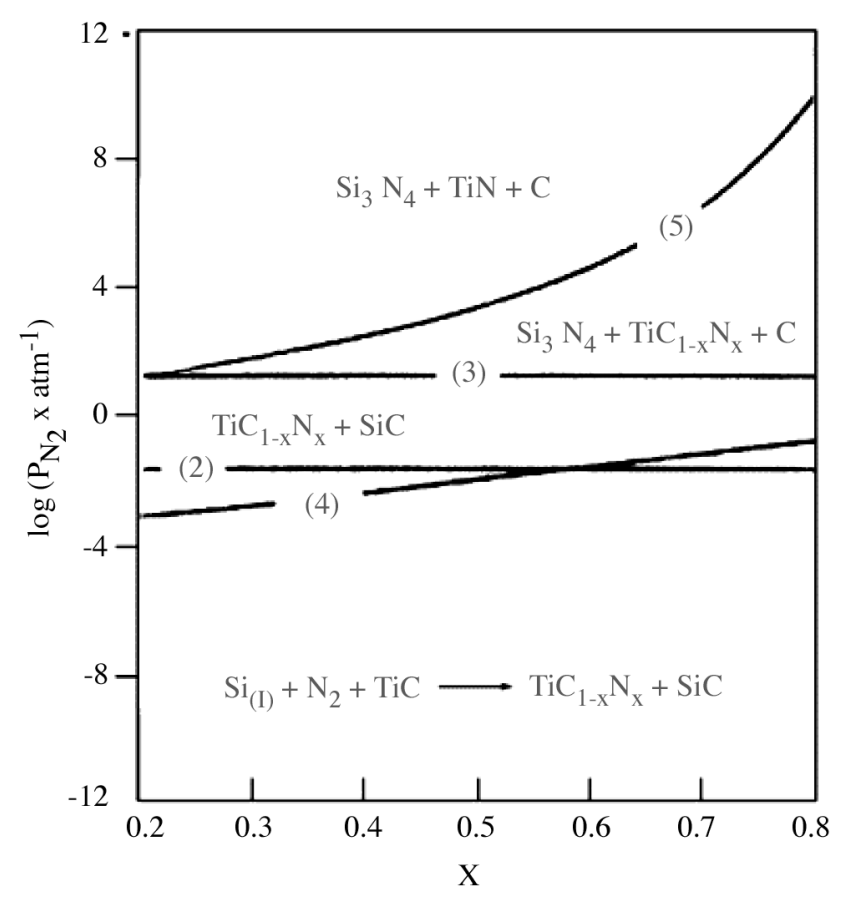

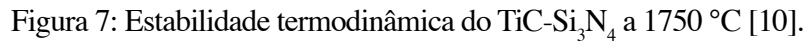

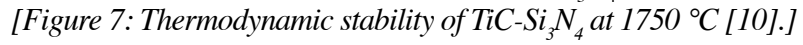

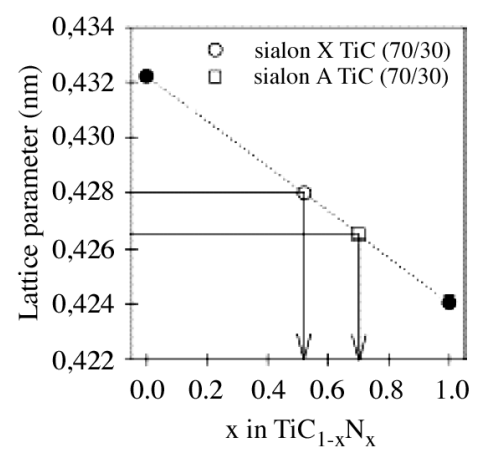

Figura 8: Parâmetro de rede do $\mathrm{TiC}_{1-\mathrm{x}} \mathrm{N}_{\mathrm{x}}$ em função de $\mathrm{x}$ [13]. [Figure 8: Lattice parameter of $\mathrm{TiC}_{1-x} N_{x}$ as a function of $x$ [13].]

A composição do carbonitreto de titânio foi determinada por difração de raios $\mathrm{X}$. As fases identificadas têm composição $\mathrm{TiC}_{0,3} \mathrm{~N}_{0,7}$ e $\mathrm{TiC}_{0,7} \mathrm{~N}_{0,3}$. $\mathrm{O}$ parâmetro de rede dessa solução sólida varia de acordo com a lei de Vegard devem ser $0,430 \mathrm{~nm}$ para a fase $\mathrm{TiC}_{0,7} \mathrm{~N}_{0,3}$ e $0,4295 \mathrm{~nm}$ para a fase $\mathrm{TiC}_{0,3} \mathrm{~N}_{0,7}$ [11] (Fig. 8).

Segundo [12] a cinética de reação para formação de TiCN é controlada por difusão. Neste caso, a reação deve ter inicio na superfície das partículas de TiC, formando uma camada de produto - TiCN. Como a difusão é um processo ativado termicamente, quanto maior a temperatura do tratamento térmico maior a espessura da camada que se forma e portanto quanto maior a temperatura do tratamento térmico maior a quantidade de TiCN formado.

A partir dos resultados obtidos no estudo das reações 
pode-se concluir que os picos observados na curva de taxa de retração linear em função da temperatura (Fig. 2 (b)) devem estar relacionados com as reações que ocorrem durante a sinterização do compósito. O pico 1 pode ser relacionado com a formação da solução sólida TiCN, o pico 2, com o processo de transformação de fase do $\mathrm{Si}_{3} \mathrm{~N}_{4}$ e o pico 3 , em temperatura mais elevada, com a formação do $\mathrm{SiC}$.

\section{CONCLUSÕES}

A presença de TiC modifica a cinética de sinterização do $\mathrm{Si}_{3} \mathrm{~N}_{4}$ e reage quimicamente com o $\mathrm{N}$, formando a fase TiCN. As alterações observadas na cinética de sinterização são causadas pelas reações químicas que ocorrem durante os tratamentos térmicos.

\section{REFERÊNCIAS}

[1] J. Blumm, Ceram. Ind. 2 (2004) 23.

[2] F. C. Pellon, F. T. Thevenot, J. Eur. Ceram. Soc. 22 (2002) 271. [3] Y. Gogotsi, J. Mater. Sci. 29 (1994) 2541.
[4] G. S. Upadhyaya, Nature and Properties of Refractory Carbides, Nova Science Publishers, Inc, (1996) p.167.

[5] Y. M. Chiang, D. Birnie III, W. D. Kingery, Physical Ceramics Principles, $1^{\text {st }}$ Ed., John Wiley \& Sons, New York (1997).

[6] S. Hampshire, K. H. Jack; in D. Taylor, P. Popper, Special Ceramics, British Ceramic Research Association, London (1981) 37.

[7] K. Negita, J. Mater. Sci. Lett. 4 (1985) 417

[8] J. F. Yang, T. Ohji, T. Sekino, C. L. Li, K. Niihara, J. Eur. Ceram. Soc. 21, 12 (2001) 2179.

[9] X. Pan, J. Mayer, M. Rühle, K. Niihara, J. Am. Ceram. Soc. 79, 3 (1996) 585.

[10] V. A. Izhevskyi, L. A. Genova, J. C. Bressiani, Mater. Res. 2, 4 (1999) 271.

[11] J. Vleugels, D. T. Jiang, O. Van Der Biest, J. Mater. Sci. 39, 7 (2004) 3375.

[12] C. H. Yeh, M. H. Hon, J. Ceram. Soc. Jpn. 102, 11 (1994) 995.

[13] H. Pastor, Mater. Sci. Eng. A 105/106 (1988) 401.

(Rec. 29/07/2004, Ac. 24/09/2004) 